\title{
CORONAVIRUS : IMPACT SUR LE TOURISME ET PRÉCAUTIONS PRÉVUES CAS DU MAROC
}

\section{J. El bordo ${ }^{1}$ M. Nadraoui ${ }^{1}$}

${ }^{1}$ Etudiant chercheur. Laboratoire: Dynamiques des Espaces et des Sociétés, Faculté des Lettres et des Sciences Humaines de Mohammedia, Hassan II University of Casablanca, Av Hassan II, B.P. 546, Mohammedia, Maroc

Professeur de géographie. Président de la bronche géographie. Laboratoire: Dynamiques des Espaces et des Sociétés, Faculté des Lettres et des Sciences Humaines de Mohammedia, Hassan II University of Casablanca, Av Hassan II, B.P. 546, Mohammedia, Maroc

Email: elbordojaoid@gmail.com

${ }^{1}$ Professeur de géographie. Président de la bronche géographie. Laboratoire: Dynamiques des Espaces et des Sociétés, Faculté des Lettres et des Sciences Humaines de Mohammedia, Hassan II University of Casablanca, Av Hassan II, B.P. 546, Mohammedia, Maroc

\section{Publié le 01/08/2021}

\section{Accepté le 24/07/2021}

\section{Résumé}

L'industrie touristique au Maroc est un secteur économique important, il possède un ensemble des opportunités touristiques qui lui ont permis d'être une destination pour les touristes du monde entier. Cependant, la propagation soudaine d'un virus appelé virus Corona a incité le Maroc à prendre un ensemble de mesures pour limiter la propagation de l'épidémie. Le secteur du tourisme a été l'un des secteurs les plus importants qui ont été touchés, a cette raison nous avons essayé de mettre en évidence, à travers cet étude, l'impact de l'apparition du Corona virus sur le tourisme et identifier les mesures les plus importantes que le gouvernement marocain a prises pour atténuer les conséquences de l'interruption de l'activité touristique.

Key Words: Corona virus ; tourisme ; interruption de l'activité touristique ; impact socio-économique. 


\title{
CORONAVIRUS: IMPACT ON TOURISM AND ANTICIPATED PRECAUTIONS \\ CASE OF MOROCCO
}

Published at 01/08/2021

Accepted at 24/07/2021

\begin{abstract}
The tourism industry in Morocco is an important economic sector, it has a set of tourism opportunities that have enabled it to be a destination for tourists from all over the world. However, the sudden spread of a virus called the Corona virus prompted Morocco to take a set of arrangements to limit the spread of the epidemic. The tourism sector has been one of the most important sectors that have been affected, for this reason we have tried to highlight, through this study, the impact of the appearance of the Corona virus on tourism and to identify the most important arrangements that the Moroccan government has taken to mitigate the consequences of the interruption of tourist activity.
\end{abstract}

Key Words: Corona virus; tourism ; interruption of tourist activity; socio-economic impact. 


\section{i. Introduction}

Le tourisme est un affluent des affluents de l'économie locale, et il est stimulé par la production et les revenus nationaux. De nombreux pays arabes, comme le Maroc, dépendent du tourisme comme affluent de l'économie. Le tourisme constitue une part importante du budget de ces pays, car ces pays imposent des visas d'entrée et de sortie ou des frais d'entrée aux lieux touristiques ce qui représente une source de revenus indispensable.

L'apparition d'une épidémie, appelée Coronavirus ou COVID-19, dans tous les pays du monde a mis en teste tous les exploits, systèmes et organisations humains. Le tourisme est l'un des secteurs qui doit affronter les conséquences de cette épidémie, puisque les flux touristiques participent à l'émergence du virus Corona.

Le Maroc, pays touristiques par excellence, a adopté des mesures urgentes pour diminuer le nombre des victimes du coronavirus, mais ses interventions à fin temps ne servent pas le secteur touristique dans le pays. Cette situation nous a poussé à faire notre recherche sur l'impact de l'apparition du "Corona virus"sur le secteur touristique Marocain, et pour cela nous avons choisis, comme axe pour cette recherche la question suivante : Quel est l'impact du coronavirus sur le tourisme au Maroc?

Nous avons procédé à plusieurs documents, bulletins administratifs et communiqués pour essayer de répondre à la question posée.

Notre étude sur était difficile en raison de notre adhésion à la quarantaine appliquée par les autorités marocaines, et malgré cela nous avons pu communiquer avec un plusieurs employés du secteur touristique ainsi que suivre les différents communiqués et bulletins ministériels sui sont disponibles sur les sites officiels.

\section{ii. Tourisme : secteur promoteur de l'économie Marocaine}

\section{Les atouts touristiques du Maroc}

De nombreux facteurs font du Maroc un pays touristique, dont les suivants:(1)

- Nature: le Maroc est la porte d'entrée du continent africain, car il possède de nombreux paysages époustouflants, tels que les sommets des montagnes; Parmi les plus importants se trouvent les sommets des montagnes du Haut Atlas, les chaînes de montagnes du Rif et se caractérisent par des oasis désertiques, des lignes côtières accidentées, des cascades et des grottes dans les collines forestières.

- Villes anciennes: Les anciennes villes marocaines sont considérées comme l'une des plus attrayantes pour les touristes, car leur construction remonte à des siècles, et il est possible de visiter la ville de Fès, le théâtre de la rue Jemaa El Fna à Marrakech et d'autres villes anciennes.

- Activités marocaines: Il existe de nombreuses activités qui peuvent être pratiquées au Maroc exclusivement, dont les suivantes:

○ Randonnées sur les plus hauts sommets d'Afrique du Nord.

- Apprenez à préparer le couscous.

- Partez en balade à dos de chameau dans le désert.

○ Promenez-vous dans les marchés du pays. 
○ Détendez-vous sur les terrasses et les places.

- Culture et traditions marocaines: Le Maroc se caractérise par la diversité de ses sectes, car ces pays ont tissé de multiples liens au fil des siècles avec: l'Afrique, l'Europe et le Moyen-Orient, et les Arabes et les Berbères ont formé un mélange réussi qui a conduit à la formation de l'identité et de la culture du pays. Histoire du Maroc: Le Maroc se distingue par sa longue histoire qui remonte à l'âge de pierre antique. (2)

\section{Lieux touristiques au Maroc}

Le Maroc est caractérisé par de nombreux lieux touristiques, dont les suivants:

- Marrakech:

Marrakech est la ville la plus populaire du Maroc, et c'est la quatrième plus grande ville du pays, et elle contient une variété de sites historiques et de régions modernes, où Il comprend deux espaces, qui sont les suivants:

-La ville: Elle se distingue par ses ruelles entrecroisées et ses boutiques d'artisanat. --Région de Glize: qui est l'opposé de la ville; Il contient des bâtiments modernes, des restaurants de restauration rapide et des restaurants urbains.

- Casablanca: C'est la plus grande ville marocaine, considérée comme la principale zone industrielle du Maroc, elle contient le quartier de la Nouvelle Médina, riche d'une charmante architecture française, et la mosquée Hassan II, qui est la plus grande mosquée d'Afrique du Nord.

- Ville de Fès: Fès est la capitale du Maroc depuis plus de 400 ans et est actuellement considérée comme le centre culturel et religieux du pays. Le centreville de Fès contient plus que de nombreuses rues étroites, qui comptent jusqu'à 9000 rues étroites, et se caractérise par ses tanneries qui valent le détour, et la ville est l'une des Sites du patrimoine mondial de l'UNESCO.

- Essaouira: C'est l'un des endroits les plus préférés des voyageurs, où vous pourrez profiter de cette ville et pratiquer de nombreuses activités, telles que: le surf, le shopping et les pique-niques sur les plages. Tanger: Tanger est l'une des plus grandes villes marocaines, car elle était d'une importance économique et culturelle sans précédent, et a été la ville de l'Empire romain pendant plus de 200 ans. (2)

- Rabat: C'est la capitale du Maroc, et la septième plus grande ville du pays, et l'un des sites patrimoniaux Monde UNESCO. (2)

\section{iii. C'est quoi le coronavirus ?}

Les coronavirus sont un grand groupe de virus qui peuvent infecter les animaux et les humains, car ils provoquent des maladies respiratoires, aussi légères que le rhume ou aussi graves que la pneumonie. Les virus Corona infectent rarement les humains puis se propagent entre eux. Et vous vous souvenez peut-être du SRAS (syndrome respiratoire aigu sévère) qui s'est propagé entre 2002-2003, qui était un exemple du coronavirus, qui a été transmis des animaux aux humains. Une autre 
nouvelle souche proéminente du virus Corona appelée MERS (syndrome respiratoire du Moyen-Orient) est apparue au Moyen-Orient en 2012, et les scientifiques disent qu'elle est initialement passée d'un chameau à un humain.

Le virus se propage généralement aux personnes par la toux, les éternuements ou le contact avec une personne infectée, ou en touchant une surface infectée, puis la bouche, le nez ou les yeux. Pour se protéger contre l'infection, lesCenters for Disease Control and Prevention (CDC) recommandent des pratiques d'hygiène de base, telles que se laver les mains fréquemment, boire fréquemment, se couvrir le nez et la bouche avec un mouchoir ou un coude en toussant et rester à la maison si vous vous sentez malade.

La grande majorité des diagnostics de virus corona équivaudra à vous informer que vous avez un rhume ou une grippe. Notez que le terme «virus corona» couvre un large éventail de virus. L'apparition d'un résultat positif lors d'une analyse de coronavirus n'est pas un sujet de préoccupation. Cela peut être très déroutant, veuillez donc travailler avec votre médecin ou votre équipe de soins si vous constatez que vous avez ce type de virus.

Les coronavirus peuvent provoquer divers symptômes, notamment de la fièvre, de la toux, un essoufflement, des maux de gorge et un écoulement nasal. La plupart des infections à coronavirus ne causent que le rhume; il existe des souches plus graves qui peuvent conduire à une pneumonie sévère qui nécessite une hospitalisation. Le CDC note que les symptômes du nouveau coronavirus comprennent «la fièvre et les symptômes qui affectent l'intérieur de la maladie respiratoire (comme la toux et la difficulté à respirer)». Les facteurs de risque comprennent le fait de voyager récemment dans certaines zones touchées ou de contacter une personne suspectée de contracter le virus.

Il n'y a pas encore de vaccin pour le traitement, et comme ce sont des virus, les antibiotiques ne fonctionneront pas avec eux. En l'absence de traitement spécifique disponible, les prestataires de soins traitent généralement les symptômes et aident le patient à se sentir plus à l'aise.

\section{iv. Tourisme et propagation du Coronavirus}

Le Maroc se distingue par une variété de qualifications touristiques, d'un riche patrimoine culturel, une situation géographique stratégique, des paysages attrayants, en plus d'une architecture touristique et hôtelière importante.En outre, d'une ville à l'autre, le royaume se trouve être un condensé de sites touristiques, avec ses belles plages et ses lieux insolites, propices à l'exploration des paysages variés du pays. Casablanca, Agadir, ou encore Marrakech, ainsi qu'une dizaine d'autres destinations marocaines sont visitées, tous les ans

Le tourisme joue un rôle important dans l'économie du pays, car il constitue l'une des principales sources de devises fortes. Il a également été en mesure de créer des emplois, de développer le secteur de la construction, de soutenir l'industrie traditionnelle et de revitaliser les villes côtières. 
Le coronavirus, au Maroc, se propage jours après joursdans toutes les régions du pays comme le montre le tableau suivant :

Répartition des cas par régions au Maroc (3)

\begin{tabular}{|l|l|}
\hline Régions & Nombre de cas confirmés \\
\hline Beni Mellal-Khénifra & 56 \\
\hline Casa Settat & 681 \\
\hline Daraa-tafilalet & 80 \\
\hline Dakhla-Oued Ed Dahab & 2 \\
\hline Fès meknes & 317 \\
\hline Guelmim Oued Noun & 1 \\
\hline Laâyoune-Sakia El Hamra & 4 \\
\hline Marrakech Safi & 487 \\
\hline Oriental & 140 \\
\hline Rabat Salé Kenitra & 258 \\
\hline Souss-Massa & 27 \\
\hline
\end{tabular}

Cette propagation a poussé le gouvernement de prendre des mesures afin de stopper ou de diminuer l'impact du Corona virus sur les citoyens au premier choix, mais cette procédure n'est pas favorable pour le secteur touristique et économique en général.

\section{v. Impact du virus sur le tourisme au Maroc et précautions prévues}

Alors que l'épidémie mondiale du virus Corona s'accélère à l'échelle national, et mondiale aussi, les crises et les conséquences de la propagation apparaissent progressivement sur le marché et l'économie, cette dernière dépend principalement de l'arrivée du tourisme étranger.

L'industrie du tourisme dans le pays est l'un des secteurs les plus importants touchés par la propagation de l'épidémie dans le monde, en particulier sur les principaux marchés du Maroc, représentés par l'Union européenne et l'Amérique, et les pays d'Asie du Sud et du Sud-est.

Selon une note publiée par CFG Bank sur l'impact du coronavirus sur le Maroc, l'économie pourra subir des impacts négatifs à cause de la pandémie du coronavirus, en particulier sur le tourisme, qui pourra subir une baisse allant jusqu'à $39 \%$ du nombre de touristes. 
Les observateurs marocains s'attendent à ce que le secteur du tourisme, qui est la deuxième source de devises étrangères, soit affecté, d'autant plus que la majorité des touristes qui visitent le pays viennent d'Europe, qui a enregistré des taux élevés d'infection par le virus.

La ville de Marrakech, comme exemple, est l'une des pôles touristiques au Maroc, cette ville reçoit un nombre intéressant de touristes chaque année et en durant toute les saisons. Le secteur touristique dans la ville de Marakech jeux un rôle très important au niveau de la mutation socio-économique de la ville elle-même et de la plus part de ses habitants ; mais après avoir enregistré deux cas de «Corona» au Pays, plusieurs manifestations, qui devaient être accueillies par la ville au cours de ce mois de mars, sont annulées suite à la publication d'un mémorandum du ministère de l'Intérieur qui interdit toutes les activités impliquant des personnes de l'étranger, y compris les séminaires Forums et toutes manifestations culturelles et sportives, ainsi que tous types de rassemblements dans des salles fermées, si le nombre de participants dépasse 1000 personnes, dans le cadre de mesures de précaution.

Bien que de nombreuses personnes qui travaillent dans le secteur touristique, avec lesquels nous nous sommes entretenus, aient estimé que cette décision porterait préjudice au secteur, qui fournit environ 400000 emplois.

le véritable problème dont souffrirait le secteur du tourisme ne serait pas la propagation du virus au Maroc, comme cela a à voir avec les pays exportant vers les touristes, notamment d'Europe (la France en particulier, qui est le premier client touristique de Marrakech) qui a averti ses citoyens De voyager vers des pays qui ne disposent pas des infrastructures et équipements sanitaires nécessaires pour soigner les cas infectés, dont le Maroc, malgré toutes les assurances données par le gouvernement.

Emergence du virus Corona au monde (4)

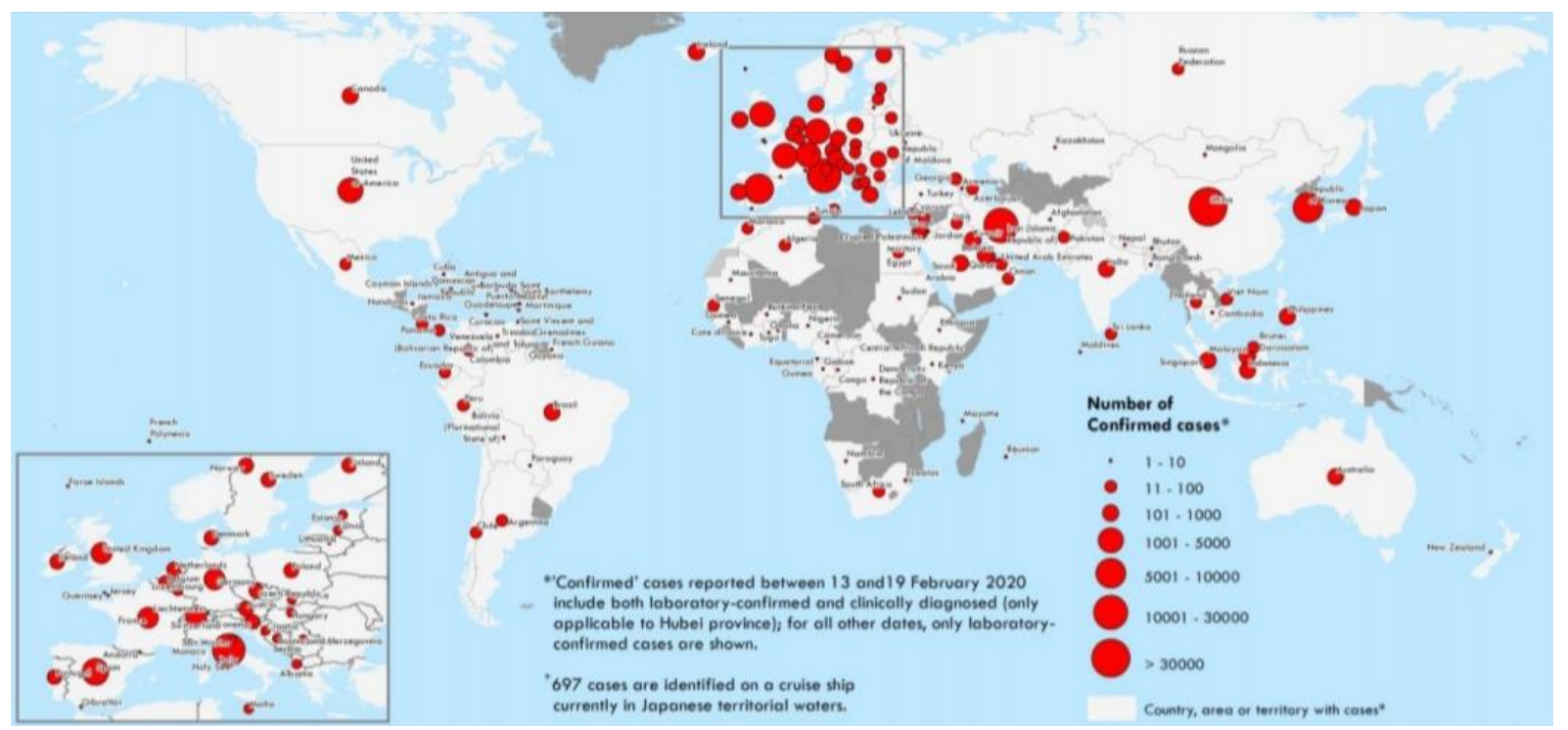

La situation mondiale est inquiétante, cependant,le Maroc a annoncé la suspension de tous les vols internationaux à destination et en provenance de son territoire, pour une durée indéterminée, afin de prévenir l'apparition du virus Corona. Un communiqué 
publié par le ministère des Affaires étrangères a déclaré: "Le Royaume a décidé de suspendre tous les vols internationaux pour transporter les voyageurs à un autre avis dans le cadre de mesures préventives contre la propagation du virus Corona".

L'apparition de quelques cas, porteurs de vertus, à l'intérieur du pays a déclenché plusieurs interventions rapide à fin de stopper l'émergence du virus. Les autorités marocaines ont décidé d'interdire l'organisation de toutes manifestations culturelles et sportives, auxquelles participent des personnes de l'étranger, ou celles de mille habitants ou plus, en cas d'établissement dans des lieux fermés, afin de lutter contre l'épidémie du coronavirus. Toutes les saisons religieuses, tels que les forums entrecoupés de symposiums et de louanges, auxquels assistait un public intéressé, seront interdits quelle que soit la taille des rassemblements auxquels il a assisté.

\section{vi. Des solutions pour éviter les effets désastreux du virus Corona sur le secteur du tourisme.}

$\mathrm{Au}$ milieu de ces évolutions rapides auxquelles le monde est témoin en raison de l'éclosion de l'épidémie de Covid 19 et de ses répercussions sur le secteur du tourisme, des solutions efficaces doivent être trouvées pour contribuer à surmonter cette crise et à atténuer son impact. À cet égard, la Confédération nationale du tourisme a proposé une série de mesures visant à aider les entreprises du secteur à surmonter les répercussions économiques du virus Corona.

Les mesures proposées par la Confédération pour accompagner le secteur face à la pandémie comprennent le report et l'annulation de l'exécution des cotisations sociales (la Caisse Nationale de Sécurité Sociale et la Caisse de Retraite Professionnelle Marocaine ...) et des exonérations fiscales (impôt sur le revenu, impôt sur les sociétés et taxe sur la valeur ajoutée ...) pendant toute la période de crise. Il est lié au report des frais bancaires de 12 mois, au maintien des lignes de crédit et à l'ouverture de lignes d'emprunt pour une période de 12 mois, tout en réduisant le taux d'intérêt à $2 \%$ pendant cette période. Parmi les mesures proposées pour suivre le rythme du secteur dans cette circonstance, On a évoqué le renforcement des garanties de prêt, la création d'un fonds pour aider les entreprises touristiques à faire face aux conséquences des crises, ainsi que la création d'un mécanisme de chômage partiel.

La Confédération nationale du tourisme a également proposé au Comité de vigilance économique que l'Etat, notamment (administration, institutions. Public ...), s'acquitte des dettes envers les acteurs et accélère le recouvrement de la taxe sur la valeur ajoutée. (5) Afin de soutenir les groupes œuvrant dans le secteur du tourisme et les travailleurs du reste des autres secteurs, un fonds spécial a été créé pour gérer la pandémie de Corona, après les hautes instructions du roi Mohammed VI, le gouvernement marocain a publié le décret $n{ }^{\circ} 2-20-269$ du 16/03/2020 pour créer un observatoire Pour les affaires privées portant la dénomination «Le Fonds de gestion de la pandémie de coronavirus « Covid-19 » (6), en plus de l'allouer comme base pour les dépenses liées à la réhabilitation des mécanismes et moyens de santé,Il contribuera également à soutenir l'économie nationale en soutenant les secteurs les plus touchés par les répercussions du Coronavirus, en préservant les emplois et en atténuant les répercussions sociales de cette pandémie. Dans le cadre de la réhabilitation du secteur du tourisme, le gouvernement a renvoyé au Parlement un projet de loi accordant aux clients une compensation et le remboursement des hôtels et des billets d'avion, en 
édictant des dispositions particulières relatives aux contrats de voyage, aux résidences touristiques et aux contrats de transport aérien pour les voyageurs. Le projet de loi vise, conformément à ses exigences, à réduire toute forme d'interruption de l'activité économique et son impact sur l'emploi; Ceci en allégeant la pression sur la trésorerie des prestataires, en insistant sur le fait d'éviter le risque de faillite des prestataires marocains et en protégeant les intérêts des créanciers, notamment des clients.

Dans ce contexte, l'article 5 du projet de loi précité prévoit que le prestataire dont les obligations découlant des contrats n'ont pas pu proposer au client au lieu de restituer les sommes payées pour les honoraires du contrat résilié et lié à une créance que ce dernier peut utiliser. À cet égard, selon le projet de loi, les propriétaires d'hôtels et d'établissements touristiques peuvent soumettre, par tous moyens, la preuve de l'arrivée dans un délai n'excédant pas 15 jours à compter de la date d'entrée en vigueur de la loi, en soulignant qu'elle indique le montant de la quittance en dette et les conditions de son utilisation. Le projet a souligné la nécessité que le service proposé soit similaire ou équivalent au service stipulé dans le premier engagement, soulignant la nécessité que le prix du service ne soit pas supérieur au prix du service stipulé dans le contrat copié et n'entraîne aucune augmentation de prix.

Les exigences de ce cadre, selon le projet gouvernemental, sont précisées pour une période précise et avec des modalités clairement énoncées, indiquant qu'elles sont liées aux contrats de voyage, aux résidences de tourisme et aux contrats de transport aérien pour les voyageurs prévus pour la période allant du 1er mars 2020 au 30 septembre 2020, qui ont été annulés en raison de l'épidémie de pandémie de coronavirus (Covid). 19 «comme une urgence difficile à prévoir et à surmonter (7). Face à cette situation difficile, une cellule de vigilance a été mise en place en lien avec le Conseil Régional du Tourisme, dans le but de sensibiliser tous les hôtels à l'importance de la mise à disposition de chambres au profit des travailleurs du secteur de la santé conformément aux diktats du principe de solidarité entre Marocains. Dans ce contexte, le Ministère du Tourisme, de l'Artisanat, du Transport Aérien et de l'Economie Sociale a révélé que les hôtels marocains ont mis gratuitement à disposition environ 13 mille chambres à la disposition des autorités gouvernementales pour contrer les effets désastreux de cette pandémie.

Le Royaume du Maroc a autorisé plus de 80000 touristes étrangers à quitter le territoire national dans le cadre du processus d'expulsion des touristes sur des vols exceptionnels, à la lumière des efforts du ministère des Affaires étrangères et de la Coopération africaine et des Marocains résidant à l'étranger. (8)

Quant aux solutions pour surmonter les défis auxquels est confronté le secteur du tourisme après la crise Corona, que la plupart des chercheurs du secteur touristique et économique jugent opportun de revitaliser le tourisme national pour compenser les pertes du tourisme étranger, et le ministère du Tourisme devrait annoncer, dans la crise post-Corona, le lancement de «cartes Voyage », avec une exonération fiscale et un soutien financier pour encourager les Marocains à ce type de tourisme domestique. 


\section{Conclusion}

En Europe, nouvel épicentre de la pandémie, les restrictions de voyage menacent la liberté de mouvement par excellence de l'Union Européenne. Au moins 10 membres de l'Union, ainsi que la Suisse et la Norvège, appliquent des contrôles aux frontières nationales - perturbant non seulement la circulation des personnes mais aussi le commerce. Du même, le Maroca pris plusieurs mesures pour réduire la gravité du virus en l'absence de vaccin ; Mais, ces mesures ont eu de nombreux effets négatifs sur l'économie du pays en général et sur le tourisme en particulier. Le tourisme au Maroc, qui a été gravement endommagé, a entraîné la perte de nombreux emploiset a montré que le pays ne s'attendait pas à faire face à de telles crises. Le fait de ne pas contenir l'épidémie de Corona dans les plus brefs délais pourrait entraîner une catastrophe économique et sociale.

Nous croyons que le moyen le plus efficace d'atténuer l'impact de cette pandémie sur le secteur du tourisme reste dépendant des efforts concertés de tous les travailleurs de ce dernier, en particulier après ces répercussions qui jetteront une ombre sur le secteur même dans l'ère post-Corona, ainsi que l'adoption de mesures sanitaires et économiques et de mesures qui restaurent fondamentalement la confiance du touriste et le protègent du facteur de peur du voyage. Le gouvernement devrait également encourager le tourisme domestique, en particulier montagneux, et réhabiliter les infrastructures pour attirer les visiteurs dans ces lieux dont le Maroc est riche, dans l'espoir de sauver ce qui peut être sauvé dans le secteur du tourisme car c'est un espace vital indispensable au tissu économique national.

\section{Bibliographie}

1- Welcome to Morocco, www.lonelyplanet.com, Retrieved 18-12-2018. Edited.

2- Khushboo Sheth (23-08-2018), "The biggest Cities In Morocco", www.worldatlas.com, retreviewed 18-12-2018

3- www.covidmaroc.ma. 17/04/2020

4- World HealtOrganization.Coronavirus disease 2019 situation report 55.15 March 2020.

4ـ مجلة 24 نيوز الإلكترونية: القطاع السياحي العالم يتوقع خسائر بقيمة 22 مليار دو لار بسبب فيروس كورونا،

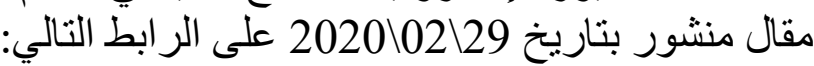

https://www.i24news.tv/ar/

5ـ مجلة منارة الخبر من الموقع الإكتروني: قطاع السياحة..اقتر اح سلسلة من التدابير لمو اجهة فيروس كورونا،

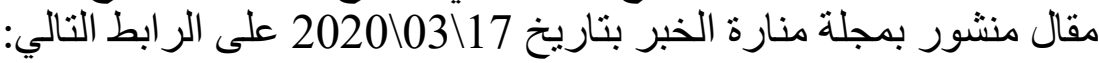

https://www.menara.ma

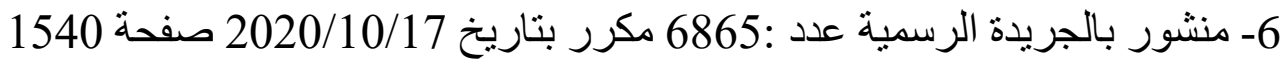
7- محمد بلقاسم : قانون يمنح الزبائن نعويض و استعادة مبلغ الفنادق،منشور بمجلة هسبريس الإلكترونية بتاريخ $2020 \backslash 5 \backslash 6$

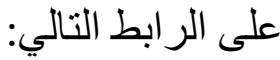

https://www.hespress.com/economie/470329.html

8- انظر مجلة سكاي نيوز عربية : كيف تعامل المغرب مع مواطنيه في المهجر، منشور بمجلة سكاي نيوز https://www.skynewsarabia.com عربية الإلكترونية بتاريخ 2029/04/26 عيدة : على الر ابط التالي: 